\title{
SPACE CHARGE EFFECT ON EMITTANCE EXCHANGE BY SKEW QUADRUPOLES
}

\author{
G. Franchetti* , I. Hofmann, GSI, Darmstadt, Germany
}

\section{Abstract}

For high current synchrotron SIS it is crucial to improve the multiturn injection efficiency. We study active emittance exchange due to a second order (skew quadrupole) difference resonance which is modified by the presence of space charge. We start from an analytical expression for the coherent tune shift of this resonance and compared it with computer simulation for the SIS synchrotron lattice including self-consistent space charge calculation. The critical issue is the ratio of incoherent space charge tune shift and resonance width. We apply the results of simulation to the horizontal multiturn injection process with space charge and find an improvement of the injection efficiency from $65 \%$ to $84 \%$ (with a correspondent increase of the vertical emittance) in a typical example.

\section{SPACE CHARGE EFFECTS IN THE LINEAR COUPLING}

One limitation of multiturn injection schemes is the loss of particles hitting the vertical septum and vertical acceptance. A skew quadrupole couples the transverse planes exciting a difference resonance. When the bare tune is on such a resonance, horizontal oscillation energy is transfered to the vertical plane and the horizontal amplitude of the injected particle will diminish during the few revolutions of the multiturn injection. This effect can be exploited to move particles away from the septum, thereby improving the injection efficiency. As reported in [1] for a single particle case, if the bare tunes are on a difference resonance $q_{x_{0}}-q_{y_{0}}=1-\delta$ with $\delta<<1$ and if the initial vertical emittance is zero, then the emittance exchange is described by the formula

$$
\epsilon_{x}=\epsilon_{x_{0}}-\frac{4 Q_{c}^{2} \sin ^{2} \Theta}{\delta^{2}+4 Q_{c}^{2}} \epsilon_{x_{0}}
$$

Here, $\Theta=2 \pi N_{t} \sqrt{\delta^{2} / 4+Q_{c}^{2}}$ and $\epsilon_{x}+\epsilon_{y}=$ const with initial values $\epsilon_{x_{0}}$ and $\epsilon_{y_{0}}=0$, where $\epsilon_{x}, \epsilon_{y}$ are the instantaneous Courant Snyder invariants, $Q_{c}=\sqrt{\beta_{x} \beta_{y}} j /(4 \pi)$, $N_{t}$ is the number of turns, and $j$ is the integrated strength of the skew. We can define the bandwidth $\delta_{b}$ of the resonance as the $\delta$ where the number of turns for a complete exchange is half of the number of turns $n_{t}$ needed for an exchange in the center of the resonance. It is straightforward to find $\delta_{b}=\sqrt{3} / n_{t}$ correspondent to the maximum exchange of $\epsilon_{x_{0}} / 4$.

\footnotetext{
*Email: G.Franchetti@gsi.de
}

\subsection{Coherent Effect}

A crucial issue is the effect of the space charge on the particle dynamics. The linear coupling produces, when the resonance condition is satisfied, a coherent effect on the beam which exchanges the two beam sizes. When space charge is present it excites a coherent tilting eigenmode with eigenfrequency $\omega$. This corresponds to $q_{x_{0}}-q_{y_{0}}$ in the absence of space charge in which case the resonance condition is $\omega=q_{x_{0}}-q_{y_{0}}=1$. Keeping $\omega=1$ as the resonance condition we can write for finite space charge by introducing a coherent tuneshift $\Delta \omega$

$$
\omega=q_{x}-q_{y}+\Delta \omega=1
$$

where $q_{x}=q_{x_{0}}-\Delta q_{x}$ and $q_{y}=q_{y_{0}}-\Delta q_{y}$ with $\Delta q_{x}, \Delta q_{y}$ the incoherent tuneshifts. By using the definitions $\sigma_{p} \equiv \omega_{p} / \nu_{x}, \alpha \equiv \nu_{y} / \nu_{x}, \eta \equiv a / b$ where $\omega_{p}$ is the "beam plasma frequency" and $a, b$ are the semiaxis of the confining ellipse, the eigenfrequency of the tilting mode is characterized by the dimensionless coherent frequency $\sigma \equiv \omega / \nu_{x}$ which results in the dispersion relation [2]

$$
\begin{aligned}
& \frac{\sigma_{p}^{2}}{2}\left(\frac{(1-\alpha)\left(1-\eta^{2} / \alpha\right)}{(1-\alpha)^{2}-\sigma^{2}}+\frac{(1+\alpha)\left(1+\eta^{2} / \alpha\right)}{(1+\alpha)^{2}-\sigma^{2}}\right)+ \\
& (1+\eta)^{2}=0
\end{aligned}
$$

We compared the prediction of the theory with simulations for the frequency of the eigenmode using for few revolutions the skew quadrupole to excite the tilting eigenmode. A coasting beam under the action of a skew kick tuned to exchange in 40 turns has been tracked for five turns and afterwards the skew was turned off to stop the exchange while the simulation continued. The coasting beam has been tracked for 274 turns, each cell of the SIS has been

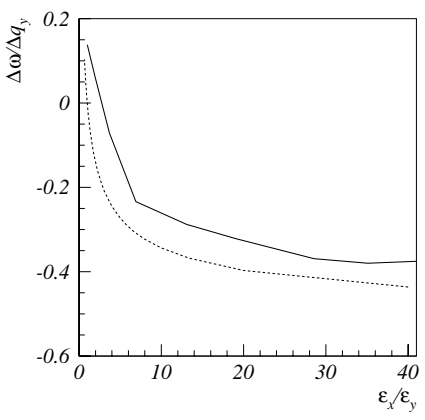

Figure 1: Comparison between analytical model (dashedline) and simulations (solid-line). 
divided in 20 steps of space charge calculation, the horizontal emittance has been kept constant to $\epsilon_{x_{0}}=200 \mathrm{~mm}$ $\mathrm{mrad}$ and the current of the beam was $I=140 \mathrm{~mA}$. At each step the quantity $\overline{x y}$, typical for the skew rotation, has been calculated and Fourier analyzed to determine $\omega$. In Fig. 1 we plot the theoretical prediction according to Eq.3 (dashed-line) and the results from simulation (solid line) for the quantity $\Delta \omega / \Delta q_{y}$ showing reasonably good agreement. In order to understand the effect of the space charge we split the eigenfrequency $\omega=\omega_{l}+\delta \omega_{s c}$ in a lattice component $\omega_{l}=q_{x_{0}}-q_{y_{0}}$ and in a space charge component $\delta \omega_{s c}=-\Delta q_{x}+\Delta q_{y}+\Delta \omega$. Analogous to the single particle theory we define the shift from the resonance as $\delta=1-\omega$. However since the space charge component $\delta \omega_{s c}$ changes according to the emittance exchange the relevant quantity which describes the dynamical regime of the beam evolution is $\delta \omega_{s c} / \delta_{b}$. Considering at the time $t=0$ $\omega=1$ we can distinguish the evolution of the beam between a weak space charge regime if $\delta \omega_{s c} / \delta_{b}<<1$ and a strong space charge regime $\delta \omega_{s c} / \delta_{b}>>1$ where the fast change of $\delta \omega_{s c}$ drives oscillations of $\delta$ up to the border of the resonance limiting the exchange. The maximum fluctuation of $\delta \omega_{s c}$ is expected to be of the order of $2 \delta_{b}$.

\subsection{Strong Space Charge Regime}

In a strong space charge regime the dynamics of the exchange is strongly nonlinear. We simulate this case for a beam with $\epsilon_{x}=195 \mathrm{~mm} \mathrm{mrad}, \epsilon_{y}=4 \mathrm{~mm} \mathrm{mrad}$ and a current $I=160 \mathrm{~mA}$. Using the results of Fig. 1, according to the previous definition, the initial space charge eigenfrequency component is $\delta \omega_{s c}=0.12$ and since $\delta_{b}=0.02$ we find $\delta \omega_{s c} / \delta_{b} \sim 6$. We expect consequently a strong deviation in the emittance exchange described by Eq. 1 . We investigated this effects repeating the same simulation with identical initial condition but different $q_{y_{0}}$, changing in this way the initial resonance condition. We distinguish between the initial eigenfrequency below the resonance $(\delta<0)$ and above the resonance $(\delta>0)$. According to the condition $\omega=1$ the center of the resonance is expected to be at $q_{y_{0}}=3.41$, but Fig. 2 shows that the exchange is strongly affected whether the resonance is reached from below or above. When the resonance is approached from below, Fig. 2b shows the existence of two regimes: one of small oscillations of $\omega$ and small exchange; and, for slightly higher working point $\omega$ sweeps through the whole stopband consistently with a much bigger emittance exchange. In this case the evolution of the exchange is not the same as described in Eq. 1 because of the dynamical change of the resonance condition. Figs. 2c-f show a slow decrease of the exchange for increasing values of $q_{y_{0}}$ that we interpret as a reduction of the swept area by $\omega$ in the bandwidth. The asymmetry of the exchange with respect to the case $\delta=0$ is the stronger the bigger is the initial ratio $\delta \omega_{s c} / \delta_{b}$. a

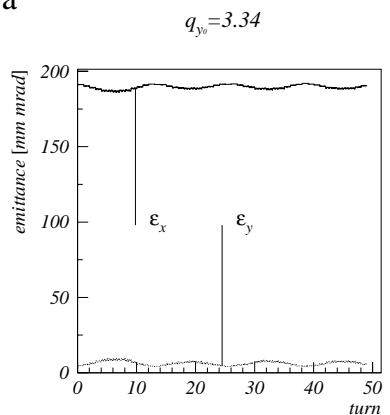

$\mathrm{c}$

$q_{y_{o}}=3.36$

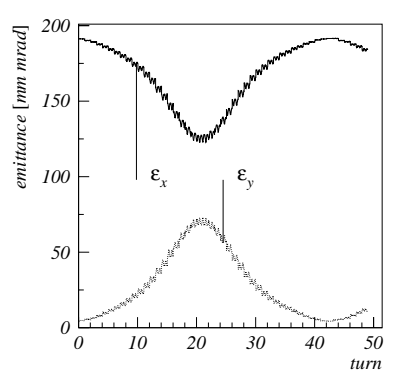

$\mathrm{e}$

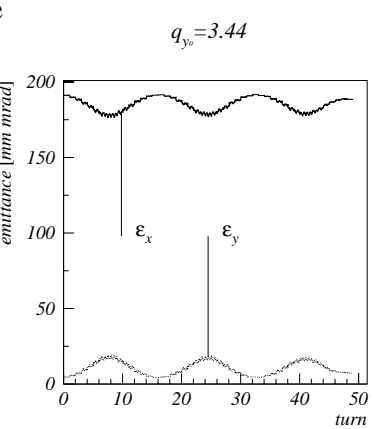

$\mathrm{b}$

$q_{y_{0}}=3.35$

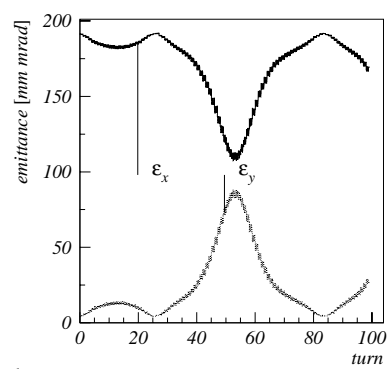

d

$q_{y_{0}}=3.39$

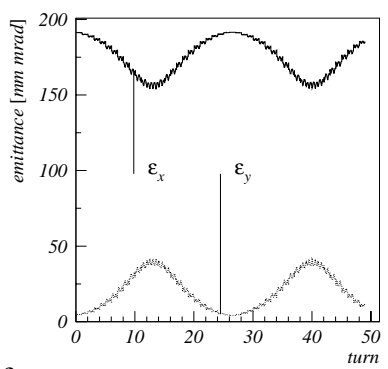

f

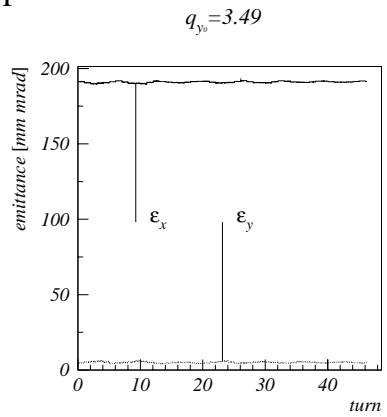

Figure 2: Strong space charge regime for $\mathrm{I}=160 \mathrm{~mA}$. a)f) show the emittance exchange for increasing $q_{y_{0}}$. b) has been tracked for 100 turns to show the deviation of the exchange from the single particle theory Eq. 1.

\section{MULTITURN INJECTION SIMULATIONS}

In this section we present a study on the multiturn injection with skew. We model the SIS injection under somewhat idealized condition assuming the injection where $\alpha_{x}=0$, reducing in such a way the septum losses. The multiturn injection scheme is modeled by a shift of the septum correspondent to the distance between septum and bumped orbit. At each turn the septum has been shifted outward by the amount $\Delta x=\sqrt{\epsilon_{x, \text { max }} \hat{\beta}_{x}} / N_{t}$, therefore its position at the $n$th turn with respect to closed orbit is $x_{\text {sept. }}=\Delta x n$. The septum scrapes all the particles satisfying the condition $x>x_{\text {sept. }}$. The acceptance is also simulated removing all the particles satisfying $x^{2} / A^{2}+y^{2} / B^{2}>1$, where $A=50.5 \mathrm{~mm}$ and $B=26 \mathrm{~mm}$. The multiturn injection takes place in 40 turns, each beamlet injected has $\epsilon_{x}=\epsilon_{y}=4 \mathrm{~mm}$ mrad with a current of $I=4 \mathrm{~mA}$. The ions used have $Z=28, A=238$ and are injected 
at $11.4 \mathrm{MeV} / \mathrm{u}$. In the simulation each beamlet is described by $N_{b}=100$ macroparticles and the space charge is calculated in a model of ellipsoidal symmetry [3]. Figs. 3a,b show the emittance increase for $q_{x_{0}}=4.29, q_{y_{0}}=3.29$ and $n_{t}=8$. Here $n_{t}$ is a dimensionless measure for the strength of the skew. It is defined as the number of turns required for a complete exchange of $\epsilon$ in the center of the resonance. Fig. 3c shows for the same setting the efficiency of the injection $N /\left(N_{b} n\right)$, the losses on the septum, and on the acceptance, ( $N$ is the number of macroparticles inside the ring). The losses up to $20 \%$ on the septum after 4 turns (change in the slope of $\epsilon_{x}$ in Fig. 3a) can be explained by the initially small distance between septum and closed orbit. After $\sim 20$ turns, due to the high strength of the skew, losses on the acceptance begin (in Fig. $3 \mathrm{~b} \epsilon_{y}$ stops its growth). At the 10th turn $I=32 \mathrm{~mA}, \delta \omega_{s c}=0.024$ which leads to the shift from the resonance of $\delta=-0.024$. By using Eq. 1 the maximum exchange is $\delta \epsilon / \epsilon_{x_{0}}=0.96$ which is obtained in 4 turns and 10th beamlet would be lost on the acceptance if $\epsilon_{x_{0}}=51$. Because $\epsilon_{x_{0}}=50 \mathrm{~mm}$ mrad the beginning of the acceptance losses at 22 turns instead of 15 must be attributed at the correction to Eq. 1 which is hold only for $\epsilon_{y_{0}}=0$. Fig. 3d shows the multiturn injection for $q_{x_{0}}=4.4$ and $n_{t}=8$. In this case space charge reduces the exchange. Let's consider again the beamlet injected after 10 turns, the closer to the resonance line which has $\epsilon_{x_{0}}=50 \mathrm{~mm}$ mrad. Repeating the previous calculation we find that it is lost if $\epsilon_{x_{0}}=105 \mathrm{~mm}$ mrad and since this emittance gap cannot be provided by the finite horizontal emittance, the acceptance losses are not present. The drawback is the increase of the septum losses since the skew is insufficient to keep the injected beamlet away from the septum. Fig. 3e shows a contour plot of the efficiency of multiturn injection for different horizontal tunes and skew strength and no space charge. We only plot the working points with integrated efficiency exceedings $65 \%$ (outer boundary), where each contour line corresponds to an increment of $1.7 \%$. Note that the efficiency region exceeding $65 \%$ disappears with $n_{t}$ exceeding 30 . The efficiency maximum of $84 \%$ is centered at $q_{x}=4.4, n_{t}=8$, where the emittance transfer horizontal to vertical just matches the vertical acceptance. Fig. 3f shows the effect of the space charge. The $84 \%$ efficiency in $q_{x}=4.4, n_{t}=7$ is close correspondent peak in Fig. 3e since the bandwidth $\delta_{b}=0.21$ is very large and $\delta \omega_{s c} \sim 0.09$, so $\delta \omega_{s c} / \delta_{b} \sim 0.42$. In $n_{t}=18$ this ratio become 1 , hence the space charge shift on the optimum working point must be taken into account. Fig. 3e suggests that the off-resonance value $\omega \sim 1.1$ is without space charge. Maintaing the same condition with space charge requires shifting $q_{x_{0}}$ by -0.1 , which agrees roughly with the estimate of section 1.1.

\section{OUTLOOK}

From the discussion on the coherent space charge effect we find that in the multiturn injection a gain up to $19 \%$ is still possible, with space charge, if an appropriate skew

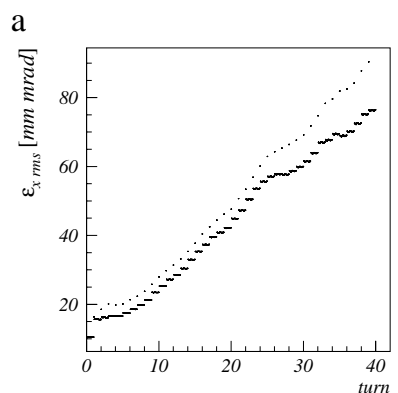

c
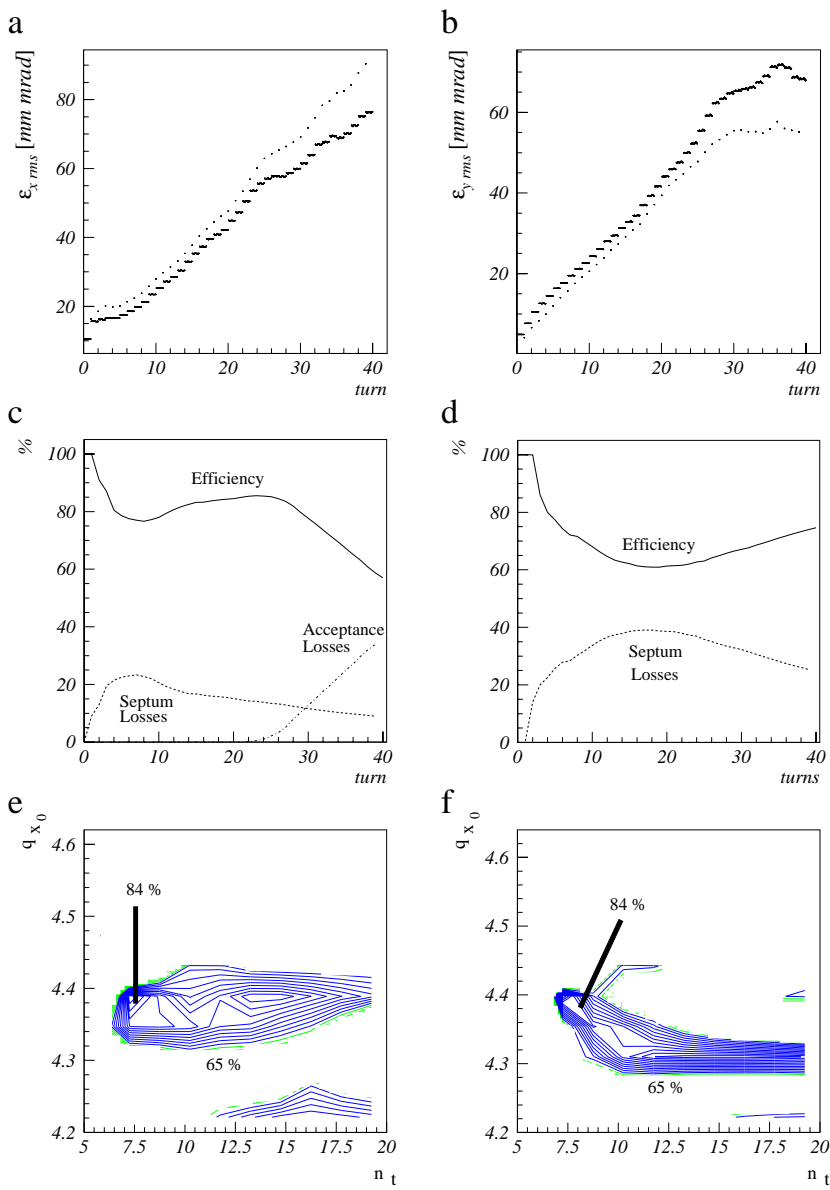

$\mathrm{d}$
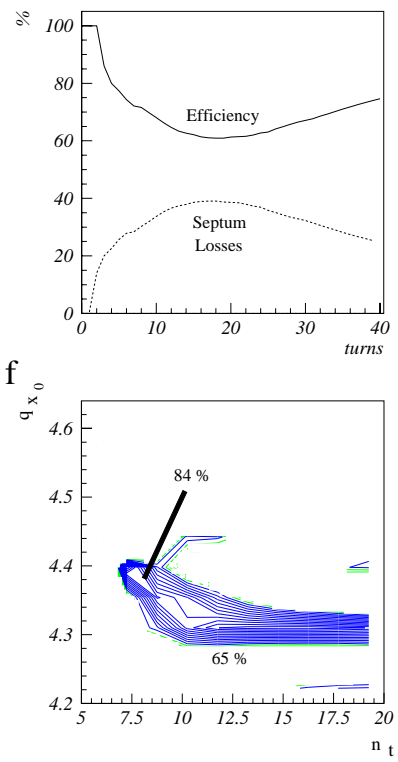

Figure 3: Multiturn injection simulations for SIS with skew quadrupole. a),b) horizontal and vertical emittance with an $q_{x_{0}}=4.29, q_{y_{0}}=3.29$ and skew strength correspondent to $n_{t}=8$; in c) injection efficiency for the same settings in a),b); d) Integrated efficiency for $q_{x_{0}}=4.4, q_{y_{0}}=3.29$ and $n_{t}=8$; e) Integrated efficiency after 40 turns in absence of space charge for $q_{y_{0}}=3.29 ; \mathrm{f}$ ) Efficiency as in e) but with space charge.

strength and shift of the working point are applied. These results should be further optimized with a more accurate space charge calculation for detailed injection conditions.

\section{REFERENCES}

[1] K. Schindl and P. Van der Stock, Increase of Betatron Stacking Efficiency via linear Coupling in AG Proton Synchrotrons ("Skew Injection"). Application to the CERN PS Booster. CERN/PS/BR 76-19.

[2] I. Hofmann, Phy. Rev. E, 57, 4713 (1998).

[3] G. Franchetti, I. Hofmann, and G. Turchetti AIP Conf. Proc. 448, 233 (1998); ed. A.U.Luccio and W.T. Weng. 